\title{
Toll-like Receptor 4 Mediates Morphine-Induced Neuroinflammation and Tolerance via Soluble Tumor Necrosis Factor Signaling
}

\author{
Lori N Eidson', Kiyoshi Inoue ${ }^{2}$, Larry J Young ${ }^{2}$, Malu G Tansey ${ }^{3}$ and Anne Z Murphy,, \\ 'Neuroscience Institute, Petit Science Center, Georgia State University, Atlanta GA, USA; ${ }^{2}$ Center for Translational Social Neuroscience, Yerkes \\ National Primate Research Center, Department of Psychiatry and Behavioral Sciences, Emory University, Atlanta, GA, USA; ${ }^{3}$ Department of \\ Physiology, Emory University, Atlanta, GA, USA
}

\begin{abstract}
Opioid tolerance and the potential for addiction is a significant burden associated with pain management, yet its precise underlying mechanism and prevention remain elusive. Immune signaling contributes to the decreased efficacy of opioids, and we recently demonstrated that Toll-like receptor 4 (TLR4)-mediated neuroinflammation in the periaqueductal gray (PAG) drives tolerance. Tumor necrosis factor (TNF), a product of TLR4 signaling, promotes inflammation and facilitates glutamatergic signaling, key components of opioid tolerance. Therefore, we hypothesize that TLR4-mediated opioid tolerance requires TNF signaling. By expression of a dominant-negative TNF peptide via lentiviral vector injection in rat PAG to sequester soluble TNF (solTNF), we demonstrate that solTNF mediates morphine tolerance induced by TLR4 signaling, stimulates neuroinflammation (increased IL- I $\beta$ and TLR4 mRNA), and disrupts glutamate reuptake (decreased GLT-I and GLAST mRNA). We further demonstrate the efficacy of the brain-permeant PEGylated version of the anti-solTNF peptide, XPro I595, injected systemically, to normalize morphine-induced CNS neuroinflammation and morphine- and endotoxin-induced changes in glutamate transport, effectively preserving the efficacy of morphine analgesia and eliminating tolerance. Our findings provide a novel pharmacological target for the prevention of opioid-induced immune signaling, tolerance, and addiction. Neuropsychopharmacology (2017) 42, 66I-670; doi: I0.1038/npp.2016.131; published online 17 August 2016
\end{abstract}

\section{INTRODUCTION}

Opioid therapy remains a common strategy for severe and chronic pain management with 3-4\% of adults in the United States receiving long-term opioid therapy (Dowell et al, 2016). However, decreased analgesic efficacy over time (ie, tolerance) significantly impedes treatment for $\sim 60 \%$ of the patient population (Gulur et al, 2014). Long-term opioid therapy is associated with increased risk of abuse, dependence, and dose-related fatal overdose (Dowell et al, 2016). Immune signaling is a significant contributor to the negative consequences of opioid therapy including tolerance, hyperalgesia, addiction, dependence, and withdrawal (Hutchinson et al, 2007), and has been implicated as a driving factor in a variety of chronic pain syndromes, including rheumatoid arthritis (RA) and fibromyalgia (Heo et al, 2011; Kosek et al, 2015). Understanding the mechanisms underlying opioidinduced neuroinflammation is paramount to developing effective pain management strategies that minimize the risk

\footnotetext{
*Correspondence: Dr AZ Murphy, Neuroscience Institute, Petit Science Center, Georgia State University, 100 Piedmont Center Avenue, SE, PO Box 40 I0, Atlanta, GA 30302, USA, Tel: +I 404413 5332, Fax: + I 404 413 530।, E-mail: amurphy@gsu.edu

Received 23 March 2016; revised 8 June 2016; accepted 10 July 20 I6; accepted article preview online 27 July 2016
}

of dependence, abuse, and long-term consequences of chronic neuroinflammation.

We recently demonstrated that chronic opioid administration in rats induces a robust neuroinflammatory response via Toll-like receptor 4 (TLR4) signaling in the periaqueductal gray (PAG), a key site for opioid-mediated analgesia, which drives tolerance (Wang et al, 2012; Eidson and Murphy, 2013). However, the mechanism by which TLR4 signaling leads to opioid tolerance is unknown. Morphine-induced TLR4 signaling increases proinflammatory cytokine production in the central nervous system (CNS) (Raghavendra et al, 2002; Shen et al, 2011a) including tumor necrosis factor (TNF). Here we test the hypothesis that TNF signaling is required for TLR4-induced tolerance.

TNF drives the release of itself and other proinflammatory cytokines (eg, IL-1 $\beta$ and IL-6) (Shen et al, 2011a, b; Sun et al, 2012), and robustly alters glutamate homeostasis and excitatory signaling in the CNS (Stellwagen et al, 2005). Interestingly, many proposed mechanisms of opioid tolerance and dependence include a role for increased glutamatergic and/or decreased GABAergic signaling (Vaughan et al, 1997; Bachtell et al, 2015), which ultimately oppose the hyperpolarizing effects of morphine. TNF naturally exists in two forms: the more common form, transmembrane TNF (tmTNF), and the less abundant form, soluble TNF (solTNF) (Kriegler et al, 1988). Although spinal TNF has been impli- 
cated in opioid tolerance maintenance (Shen et al, 2011a), TNF has not been examined in isolation in the development of opioid tolerance, and it is not known which form of TNF mediates tolerance development and glutamatergic signaling.

The present studies tested the hypothesis that TLR4 elicits opioid tolerance via solTNF-mediated increases in PAG immune signaling and disruption of glutamate reuptake. To test this hypothesis, we manipulated PAG solTNF using a lentivirus encoding dominant-negative TNF (DN-TNF) or XPro1595, a PEGylated brain-permeant peptide, to sequester solTNF and examined the impact on tolerance, cytokine expression, and key elements of glutamate homeostasis. Our results demonstrate for the first time that the solTNF (and not tmTNF) increases cytokine expression and alters glutamate homeostasis within the PAG, providing a potential mechanism driving the development of opioid tolerance. Taken together, these results suggest a novel pharmacological target to enhance the efficacy of opioid analgesia in the clinic while minimizing the risk of dependence and addiction.

\section{MATERIALS AND METHODS}

\section{Subjects}

Male Sprague-Dawley rats (250-350 g; Charles River, MA) were pair-housed on a 12:12 hour light-dark cycle (lights on at 0700 hours) with ad libitum access to food and water. The Institutional Animal Care and Use Committee at Georgia State University approved all studies.

\section{Lentiviral DN-TNF}

The human full-length DN-TNF DNA sequence (TNF variant with two point mutations; A145R/I97T; Steed et al, 2003) and the enhanced green fluorescent protein DNA sequence (green fluorescent protein (GFP); reporter) were subcloned into a lentiviral vector under the control of the chicken $\beta$-actin cytomegalovirus (CAG) promoter as published previously (McCoy et al, 2008). Details of lentiviral production and analysis of expression following behavioral testing are described in Supplementary Information.

\section{Lentivirus Infection and Morphine Treatment}

Bilateral intra-ventrolateral PAG (vlPAG) infusion of lentiDN-TNF or lenti-GFP were performed as described in Supplementary Information. After 1 week, morphine $(5 \mathrm{mg} /$ $\mathrm{kg}$, subcutaneously; National Institute on Drug Abuse, Bethesda, MD) or vehicle (saline; $1 \mathrm{ml} / \mathrm{kg}$, subcutaneously) was administered once a day for 3 consecutive days, resulting in four groups: lenti-DN-TNF+Morphine $(n=10)$, lenti-DNTNF+Saline $(n=5)$, lenti-GFP+Morphine $(n=10)$, and lenti-GFP+Saline $(n=4)$.

\section{Cannulation, Lentivirus Infection, and LPS Microinfusions}

Rats were implanted with bilateral cannula aimed at the vlPAG and received bilateral infusions of lenti-DN-TNF or lenti-GFP while under surgical anesthesia as described in the Supplementary Information. One week following viral vector infusion, cannulated rats received daily bilateral intra-vlPAG lipopolysaccharide or saline for 3 days to induce a naïve morphine tolerance, resulting in the following groups: lentiDN-TNF+PAG LPS $(n=9)$, lenti-DN-TNF+PAG Saline $(n=6)$, lenti-GFP+PAG LPS $(n=4)$, and lenti-GFP+PAG Saline $(n=6)$. See Supplementary Information for details.

\section{XPro1595 and Morphine Treatment}

XPro1595 (10 mg/kg; subcutaneously), a brain permeant TNF variant that selectively inhibits solTNF signaling (>2500-fold) (Steed et al, 2003; Zalevsky et al, 2007), or saline was administered 1 day before the first morphine ( $5 \mathrm{mg} / \mathrm{kg}$; subcutaneously) injection and with the final (third) morphine injection (half-life $=18 \mathrm{~h}$ ), resulting in the following groups: XPro+Morphine $(n=11)$, XPro+Saline $(n=8)$, Vehicle+Morphine $(n=11)$, and Vehicle+Saline $(n=8)$.

\section{Behavioral Testing}

Nociception (paw withdrawal latency) was assessed using the paw thermal stimulator as described in detail in the Supplementary Information (Hargreaves et al, 1988).

\section{In Situ Hybridization Analysis of the Astrocytic Glutamate Transporters GLAST and GLT-1, and the Neuronal Excitatory Amino-Acid Carrier EAAC1}

In a subset of rats ( $n=4-6$ per group), in situ hybridization for GLAST, GLT-1, and the neuronal excitatory amino acid carrier 1 (EAAC1) mRNA was performed using ${ }^{35}$ S-UTP-labeled RNA probes generated from cloned fragments of cDNA derived from rat brain tissue as described previously (Inoue et al, 2004). Details are provided in Supplementary Information. Changes in mRNA were assessed using ANOVA, and Fisher's post hoc tests when a significant main effect was observed (SPSS). Mean specific hybridization is reported as the disintegrations per minute per milligram of tissue (dpm/ $\mathrm{mg}) \pm$ S.E.M.; $p<0.05$ was considered significant.

\section{XPro1595 Measurement}

Immediately following tolerance assessment, cerebrospinal fluid (CSF) was collected via cisterna magna, and plasma and brains were collected (in under $2 \mathrm{~min}$ ). Midbrain, CSF, and plasma XPro1595 was quantified as described in Supplementary Information.

\section{qPCR Analysis of Proinflammatory Cytokines and TLR4 mRNA}

Brains were collected from rats $(n=5$ per group treated with XPro+Morphine, XPro+Saline, Vehicle+Morphine, and Vehicle+Saline, blocked (bregma -6.96 to -8.52 ), and $1 \mathrm{~mm}$ bilateral micropunches were taken through the vlPAG for quantitative RT-PCR of proinflammatory cytokines and TLR4 mRNA as described in Supplementary Information.

Gene expression is reported as the ratio of the gene of interest to Gapdh, and is normalized to the Vehicle+Saline condition. Changes in mRNA between groups were assessed using an ANOVA on ranks (Kruskal-Wallis), and MannWhitney $U$-test post hoc comparisons (SPSS). Data are expressed as median; $p<0.05$ was considered significant. 


\section{RESULTS}

\section{Sequestration of vlPAG SolTNF Eliminated Tolerance to Systemic Morphine}

Our initial series of experiments determined if sequestration of solTNF attenuated the development of tolerance to systemic morphine. The DN-TNF rapidly forms heterotrimers with native solTNF, effectively sequestering endogenous solTNF and inhibiting TNF receptor 1 (TNFRI) binding (Steed et al, 2003), while sparing tmTNF signaling (Steed et al, 2003; Zalevsky et al, 2007). Human DN-TNF and GFP or GFP alone was bilaterally expressed in the vlPAG of adult male rats via lentiviral infection 7 days before systemic morphine $\left(5 \mathrm{mg} / \mathrm{kg}\right.$; $\mathrm{ED}_{50}$ dose; Loyd et al, 2008) or saline $(1 \mathrm{ml} / \mathrm{kg})$, once daily for 3 consecutive days to induce tolerance.

Robust virus-induced GFP expression (Figure 1a) was observed bilaterally in the vlPAG as indicated by fluorescein isothiocyanate (FITC) microscopy (Figure 1c). Baseline and postinjection paw withdrawal latencies did not differ between lenti-DN-TNF and lenti-GFP rats treated with systemic saline, indicating that DN-TNF expression did not alter basal nociceptive thresholds (Figure 1b, black symbols). Administration of morphine on Days 1 and 3 produced an increase in both lenti-DN-TNF-treated rats and lenti-GFP controls (red symbols). Two-way ANOVA revealed a significant interaction between solTNF sequestration and morphine efficacy across time, $\mathrm{F}_{(9,54)}=23.51 ; p<0.0001$. Morphine efficacy did not differ between lenti-DN-
TNF+Morphine and lenti-GFP+Morphine groups on Day 1 (first morphine injection; $p>0.05$ ). On Day 3, morphine was significantly more efficacious in lenti-DN-TNF+Morphinetreated rats compared with that in lenti-GFP+Morphinetreated rats (third morphine injection; $p<0.0001$ ) indicative of tolerance only in the lenti-GFP+Morphine group.

Administration of cumulative doses of morphine on Day 4 produced an increase in paw withdrawal latency in all rats tested (Figure 1d; $\mathrm{F}_{(3,195)}=49.64, p<0.0001$ ). Post hoc analysis revealed that lenti-GFP+Morphine-treated rats were tolerant to morphine, as indicated by a threefold rightward shift in the dose-response curve $\left(\mathrm{ED}_{50}=7.47 \mathrm{mg} / \mathrm{kg}\right.$, as compared with lenti-GFP+Saline controls, $\mathrm{ED}_{50}=2.50 \mathrm{mg} / \mathrm{kg}$; $p=0.01$; Supplementary Table 1). Intra-vlPAG pretreatment with lenti-DN-TNF preserved the antinociceptive potency of morphine $\left(\mathrm{ED}_{50}=2.66 \mathrm{mg} / \mathrm{kg}\right)$ compared with rats made tolerant to morphine (lenti-DN-TNF+Morphine $v s$ lenti-GFP+Morphine; $p=0.01$ ). Indeed, lenti-DN-TNF + Morphine-treated rats did not differ from rats who received saline before the morphine challenge $(p=0.15)$.

\section{Sequestration of vlPAG SolTNF Eliminated Morphine Tolerance Induced by vlPAG LPS}

To test our hypothesis that morphine acts through TLR4 to increase solTNF signaling and decrease morphine efficacy, we repeated the above experiment in rats that received intravlPAG infusions of the prototypical TLR4 agonist LPS for 3 days to induce a naïve tolerance to morphine. Robust a
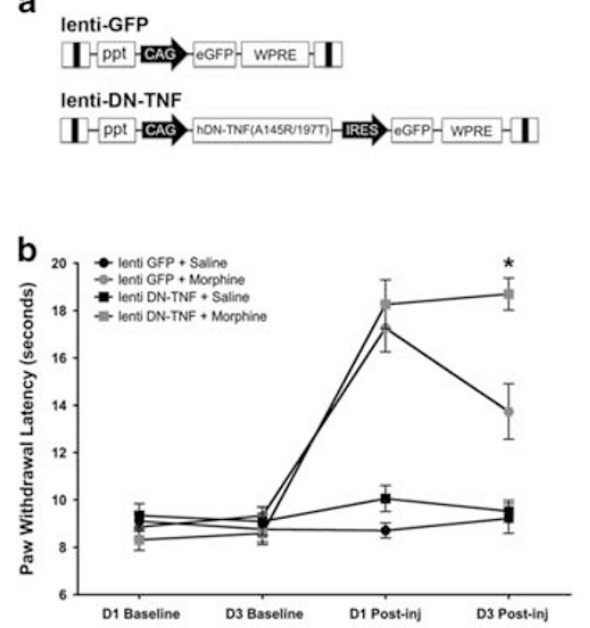

c
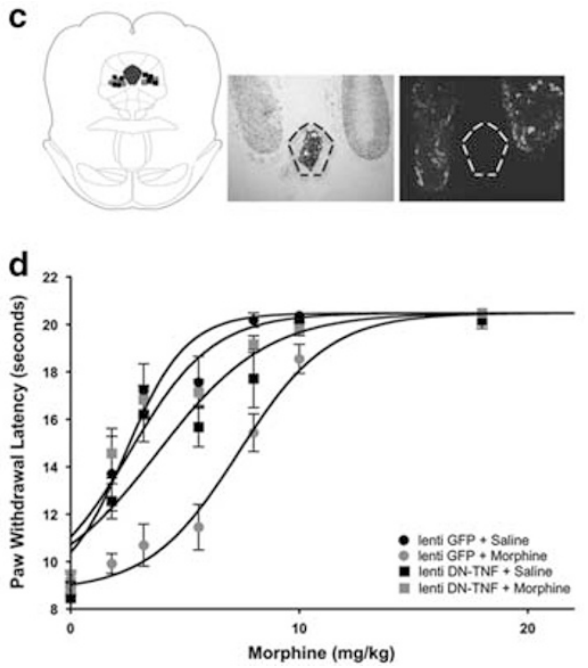

e
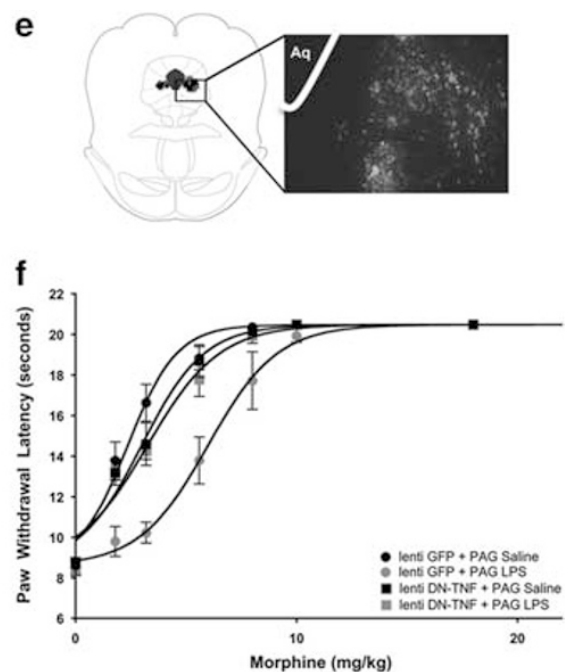

Figure I Viral vector-mediated sequestration of soluble tumor necrosis factor (TNF) in the ventrolateral periaqueductal gray (VIPAG) prevents the development of tolerance to systemic morphine and to lipopolysaccharide (LPS) microinfusion into the vIPAG. (a) Schematic of lenti-GFP and lenti-DN-TNF lentiviral vectors. Both lenti-GFP and lenti-DN-TNF vectors contain a chicken $\beta$-actin cytomegalovirus enhancer/promoter (CAG), $\beta$-globin intron, a central polypurine tract of HIV-I (ppt), a woodchuck hepatitis virus post-transcriptional response element (WPRE), and a self-inactivating deletion in the 3'-long terminal repeat (3'-LTR). The lenti-DN-TNF vector contains an internal ribosome entry site for GFP expression following the sequence for pro-human DNTNF (AI45R/I97T). (b) Paw withdrawal latency (s) during nociceptive testing on day I (DI) and day 3 (D3) at baseline and I5 min postsaline or morphine injection in groups pretreated with lenti-GFP or lenti-DN-TNF; Lenti-GFP+Saline ( $n=4$; black circles), lenti-DN-TNF+Saline ( $n=5$; black squares) lenti-GFP +Morphine ( $n=10$; red circles), and lenti-DN-TNF+Morphine ( $n=10$; red squares). (c) Representative locations of lenti-GFP (circles) and lenti-DN-TNF (squares) microinfusions in the caudal VIPAG (bregma -8.03), and representative light and fluorescein isothiocyanate (FITC) images of injection location and GFP expression from the same section. Dotted lines delineate cerebral aqueduct. (d) Paw withdrawal latency (s) during cumulative morphine injections on day 4 to assess for tolerance (see Supplementary Table I for ED ${ }_{50}$ s). (e) Representative locations of lenti-GFP (circles) and lenti-DN-TNF (squares) microinfusions in the VIPAG (bregma - 8.04), and representative FITC photomicrograph of GFP expression in the VIPAG. Aq indicates the location of the aqueduct. (f) Paw withdrawal latency $(s)$ during cumulative morphine injections in rats pretreated with lenti-GFP+PAG Saline $(n=4$; black circles), lenti-GFP+PAG LPS ( $n=4$; red circles), lenti-DN-TNF+PAG Saline ( $n=6$; black squares), and lenti-DN-TNF+PAG LPS $(n=9$; red squares). $* p<0.000$ I. DN-TNF, dominant-negative TNF; ED 50 , effective dose 50\%; GFP, green fluorescent protein. A full color version of this figure is available at the Neuropsychopharmacology journal online. 
virus-induced GFP expression was observed bilaterally in the vlPAG (Figure 1e). Consistent with our previous study (Eidson and Murphy, 2013), intra-PAG administration of LPS had no effect on baseline paw withdrawal latencies when measured 24 and $72 \mathrm{~h}$ later (data not shown). Administration of cumulative doses of morphine on Day 4 (first morphine exposure) produced an increase in paw withdrawal latency in all rats tested (Figure 1f; significant main effect of treatment: $\left.\mathrm{F}_{(3,167)}=37.31, p<0.001\right)$. Three intra-vlPAG infusions of LPS was sufficient to induce 'naïve' tolerance to morphine, as indicated by a rightward shift in the morphine doseresponse curve (lenti-GFP+PAG LPS, $\mathrm{ED}_{50}=6.00 \mathrm{mg} / \mathrm{kg}$ ) as compared with lenti-GFP+PAG Saline controls $\left(\mathrm{ED}_{50}=2.37 \mathrm{mg} / \mathrm{kg} ; \quad p=0.02\right.$; Supplementary Table 1$)$. Intra-vlPAG pretreatment with lenti-DN-TNF preserved the antinociceptive potency of morphine (lenti-DN-TNF + PAG LPS, $\mathrm{ED}_{50}=3.26 \mathrm{mg} / \mathrm{kg}$ ) compared with rats made tolerant to morphine by vIPAG LPS (lenti-GFP+PAG LPS; $p=0.01)$. Indeed, lenti-DN-TNF+PAG LPS rats did not differ from lenti-DN-TNF+PAG Saline controls $(p=0.07)$. These results demonstrate that LPS mimics the effects of morphine, acting through TLR4 to increase solTNF signaling and decrease morphine efficacy (ie, induce tolerance).

\section{Chronic Systemic Morphine Decreased vlPAG GLT-1 and GLAST mRNA in a solTNF-Dependent Manner}

We next tested the hypothesis that solTNF contributes to tolerance development by decreasing astrocytic GLT-1 and GLAST, the primary source of glutamate reuptake in the brain (60-80\%; Rothstein et al, 1996); changes in the neuronal glutamate transporter EAAC1 were also investigated. mRNA in the caudal vlPAG of rats treated with lenti-GFP+Morphine, lenti-DN-TNF+Morphine, lenti-GFP+ Saline, and lenti-DN-TNF+Saline were determined using in situ hybridization. Morphine administration for 3 days significantly decreased vlPAG GLT-1 mRNA, as compared with saline controls (main effect of treatment: $\mathrm{F}_{(3,23)}=3.58, \quad p=0.03$; lenti-GFP+Morphine $v s$ lenti-GFP + Saline; $p=0.004$; Figure 2a). Lentiviral expression of DNTNF in the vlPAG rescued the expression of GLT-1 mRNA, such that lenti-DN-TNF+Morphine rats did not differ from lenti-GFP+Saline controls $(p=0.13)$. Similar to what was noted for GLT-1 mRNA, chronic morphine significantly decreased vlPAG GLAST mRNA as compared with saline controls (main effect of treatment: $\left(\mathrm{F}_{(3,26)}=6.681, p=0.002\right.$; lenti-GFP+Morphine $v s$ lenti-GFP+Saline; $p<0.001$; Figure 2c). vlPAG DN-TNF expression prevented morphineinduced decrease in vlPAG GLAST mRNA, as lenti-DN-TNF +Morphine rats did not differ from lenti-GFP+Saline controls $(p=0.49)$. No significant effect of treatment was noted in vlPAG EAAC1 mRNA expression $\left(\mathrm{F}_{(3,26)}=0.876\right.$, $p=0.47$; Figure $2 \mathrm{e})$, suggesting that morphine preferentially alters astrocytic, and not neuronal, glutamate transport in the vIPAG.

\section{Chronic vlPAG TLR4 Agonism Decreased vlPAG GLT-1 and GLAST mRNA in a solTNF-Dependent Manner}

Given that LPS binding to TLR4 is a potent inducer of TNF expression, we hypothesized that GLT-1 and GLAST would be decreased in rats made tolerant to morphine by LPS (naïve tolerance). Consistent with our results from chronic systemic morphine exposure, chronic vlPAG microinfusions of LPS decreased vlPAG GLT-1 and GLAST mRNA $\left(\mathrm{F}_{(3,12)}=7.268, p=0.01\right.$ and $\mathrm{F}_{(3,10)}=7.321, p=0.01$, respectively), and did not alter vlPAG EAAC1 mRNA $\left(\mathrm{F}_{(3,10)}=\right.$ 1.982, $p=0.17$; Figure 2b, d, and f). Lenti-GFP+PAG LPS rats had significant decreases in GLT-1 and GLAST mRNA as compared with lenti-GFP+PAG Saline rats $(p=0.005$ (Figures $2 \mathrm{~b}$ ) and $p=0.002$ (Figure 2d), respectively). vlPAG DN-TNF expression rescued GLT-1 mRNA (lenti-DN-TNF + PAG LPS vs lenti-GFP+PAG Saline; $p=0.72$ ), and increased, but did not rescue, GLAST mRNA (lenti-DNTNF+PAG LPS $v$ s lenti-GFP+PAG Saline; $p=0.04$ ).

\section{Systemic Administration of the SolTNF Inhibitor XPro1595 Prevented Morphine Tolerance}

We next tested the effect of systemic XPro1595 on morphine tolerance using the same paradigm as above. XPro1595 (10 mg/kg; subcutaneously) or vehicle (saline; $1 \mathrm{ml} / \mathrm{kg}$; subcutaneously) was administered 1 day before the first morphine/saline injection, and with the third morphine/ saline injection. Two-way ANOVA revealed a significant interaction between solTNF sequestration and morphine efficacy across time, $\mathrm{F}_{(9,42)}=14.15 ; p<0.0001$ (Figure 3a). Morphine efficacy did not differ between rats treated with XPro1595+Morphine and those treated with Vehicle+Morphine on Day 1 (first morphine injection; $p>0.05$ ). On Day 3, morphine was significantly more efficacious in XPro1595 +Morphine-treated rats compared with that in Vehicle + Morphine-treated rats $(p<0.001)$. Administration of cumulative doses of morphine on Day 4 produced an increase in nociceptive thresholds in all rats tested (Figure 3b; $\left.\mathrm{F}_{(3,258)}=87.31, p<0.0001\right)$. Vehicle+Morphine-treated rats were tolerant to morphine $\left(\mathrm{ED}_{50}=8.03 \mathrm{mg} / \mathrm{kg}\right)$, in comparison with Vehicle+Saline rats $\left(\mathrm{ED}_{50}=2.31 \mathrm{mg} / \mathrm{kg} ; p<0.001\right)$. Systemic pretreatment with XPro1595 preserved the antinociceptive potency of morphine (XPro+Morphine; $\mathrm{ED}_{50}=$ $3.08 \mathrm{mg} / \mathrm{kg}$ ) compared with vehicle-treated rats that were tolerant to morphine (Vehicle+Morphine; $p<0.001$ ). An ELISA for human TNF (hTNF) revealed robust XPro1595 levels in plasma, CSF, and midbrain tissue (Supplementary Figure 1). Indeed, midbrain levels strongly correlated with CSF levels of hTNF $\left(R^{2}=0.87\right)$, indicating efficient transport into the brain. hTNF was not detected in Vehicle+Morphineor Vehicle+Saline-treated rats (data not shown).

\section{Systemic Administration of XPro1595 Abolished Morphine-Induced Increases in vIPAG TLR4 and IL-1 $\beta$ mRNA}

TNF and TLR4 are major regulators of cytokine expression in the CNS (eg, IL-6, IL-1 $\beta$, and TNF), and cytokines, including IL-1 $\beta$ and IL- 6 , have been implicated in morphine tolerance. We tested the hypothesis that chronic morphine increases proinflammatory cytokine expression in the vlPAG in a solTNF-dependent manner. Changes in vlPAG TLR4 mRNA were also assayed. qPCR revealed that morphine significantly increased vlPAG TLR4 expression $(H=7.49$, 2 d.f., $p=0.02$; Figure $4 \mathrm{a}$ ), such that TLR4 gene expression significantly increased in Vehicle+Morphine-treated rats as compared with XPro+Saline controls ( $W=15 ; p=0.02)$. 

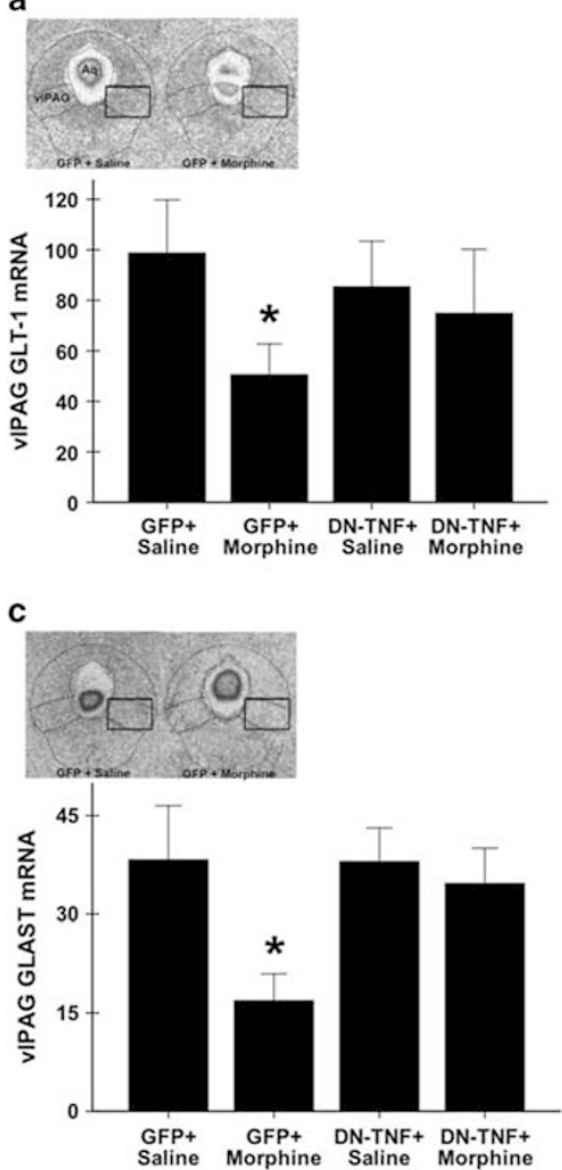

e

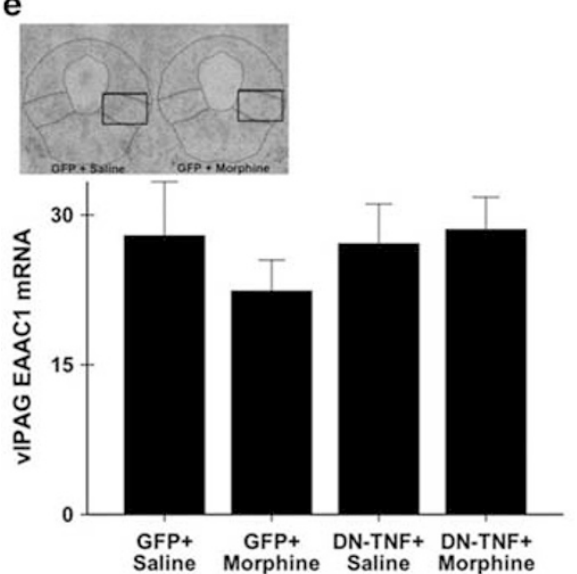

b

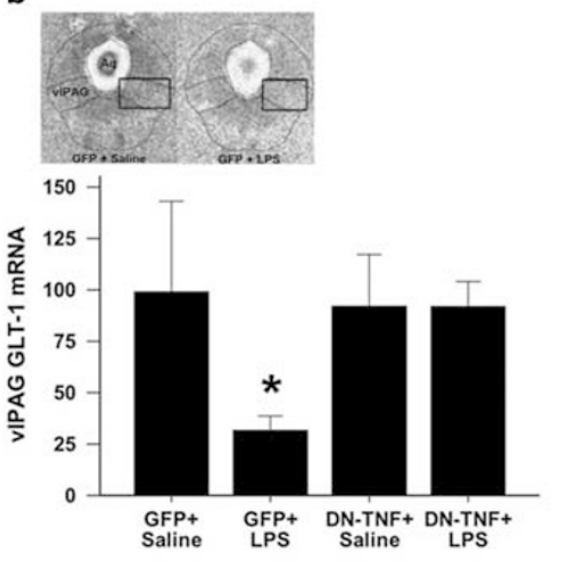

d

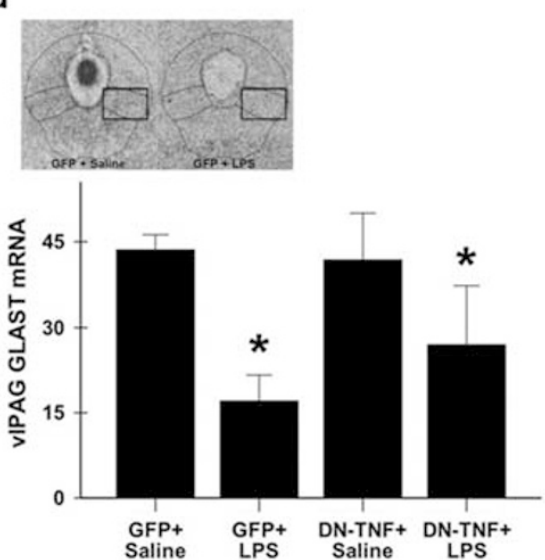

f
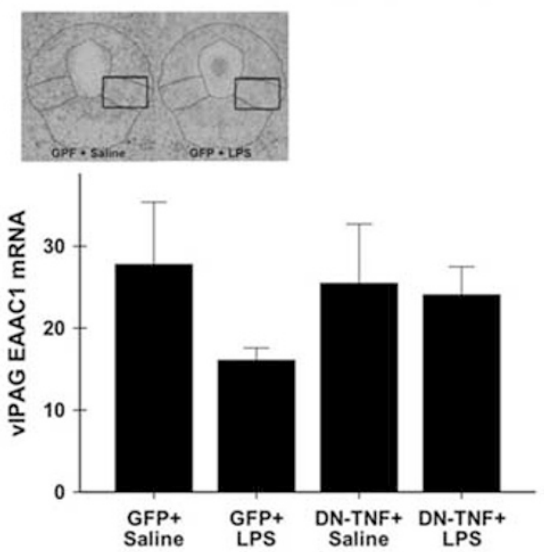

Figure 2 Viral vector-mediated sequestration of soluble tumor necrosis factor (TNF) in the ventrolateral periaqueductal gray (vIPAG) attenuates the systemic morphine- and vIPAG lipopolysaccharide (LPS)-induced decrease in astrocytic glutamate transporter mRNA in the vIPAG. Representative photomicrographs (left inset) of specific hybridization in the caudal PAG (bregma - 8.04) and bar graph of mean specific hybridization signal for (a) glutamate type I transporter (GLT-I), (c) glutamate aspartate transporter (GLAST), and (e) neuronal excitatory amino-acid carrier I (EAACI) in the caudal vIPAG of rats treated with lenti-GFP+Saline and lenti-GFP+Morphine (right). Representative photomicrographs (left inset) of specific hybridization and bar graph showing mean specific hybridization for (b) GLT-I, (d) GLAST, and ( $f$ ) neuronal EAACI in the caudal PAG (bregma - 8.04) of rats treated with lenti-GFP+PAG Saline (left) and lenti-GFP+PAG LPS (right). Specific hybridization is reported as the mean disintegrations per minute per milligram of tissue (dpm/mg) \pm S.E.M.; $n=4 / 6$ per group). *indicates a significant difference from the GFP + Saline group; * $p<0.05$. Aq delineates cerebral aqueduct and the black box represents the sampling region. GFP, green fluorescent protein.

Coadministration of XPro1595 with morphine normalized TLR4 mRNA as XPro+Morphine and XPro+Saline rats did not differ $(W=17.5, p=0.06)$. A significant main effect of treatment was also observed for vlPAG IL-1 $\beta$ mRNA by
$3500 \%$ (Kruskal-Wallis, $H=8.05,2$ d.f., $p=0.02$; Figure $4 \mathrm{~b}$ ) as compared with Vehicle+Saline controls, a nearly 30 -fold increase. Coadministration of systemic XPro1595 and morphine eliminated the increase in IL-1 $\beta$ gene expression 
666
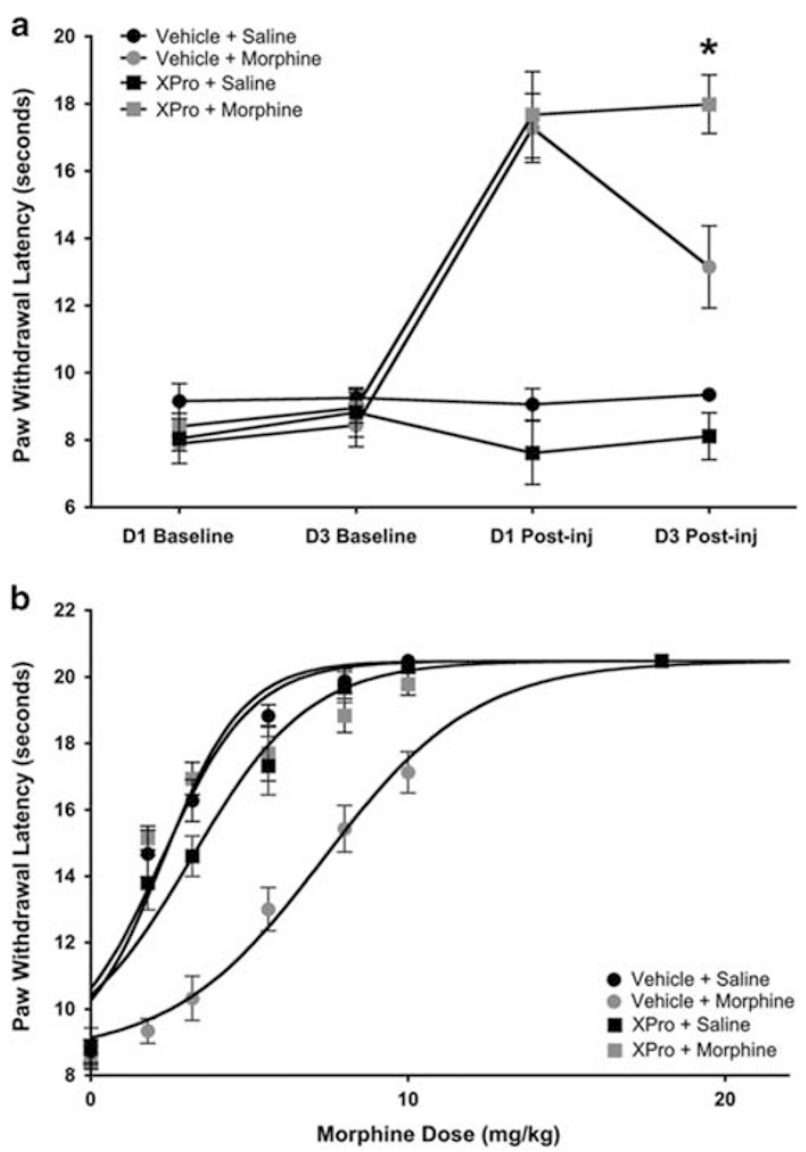

Figure 3 Summary of acute morphine analgesia and morphine tolerance following systemic XProl595 and morphine. Systemic administration of XProl595 prevents the development of tolerance to morphine. (a) Paw withdrawal latency (s) during nociceptive testing on day I (DI) and day 3 (D3) at baseline and 15 min postsaline or morphine injection in groups pretreated with XPro 595 or Vehicle (saline); Vehicle+Saline $(n=8$; black circles), XPro+Saline ( $n=8$; black squares), Vehicle+Morphine $(n=11$; red circles), and XPro I595+Morphine ( $n=1 \mathrm{I}$; red squares). (b) Paw withdrawal latency (s) during cumulative morphine injections on day 4 (see Table I for $\left.E_{50} \mathrm{~S}\right)$. Data are represented as mean paw withdrawal latency \pm S.E.M. $* p<0.000$ I. ED 50 , effective dose $50 \%$. A full color version of this figure is available at the Neuropsychopharmacology journal online.

( $W=26 ; p=0.84)$. The increase in TNF gene expression was not statistically significant (Kruskal-Wallis, $H=4.00,2$ d.f., $p=0.14)$. No significant change in IL-6 or IL-10 mRNA was noted.

\section{DISCUSSION}

\section{TLR4 Signaling Contributes to the Adverse Consequences of Chronic Opioid Use}

Chronic morphine administration leads to the induction of a neuroinflammatory response (Eidson and Murphy, 2013), resulting in a significant increase in glutamatergic tone and excitatory neurotransmission (Fine et al, 1996; Ogoshi et al, 2005), which together actively oppose the analgesic effect of morphine (Trujillo and Akil, 1991; Wong et al, 2002). These same characteristics underlie opioid dependence, withdrawal, and addiction, implicating a common underlying mechanism (Hutchinson et al, 2007). We recently demonstrated that PAG TLR4 is necessary for the development of morphine tolerance, and there is a growing body of literature implicating TLR4 in opioid reward and reinforcement (Bachtell et al, 2015). TLR4 binds opioids, including morphine, resulting in the production of a milieu of proinflammatory signaling factors (eg, TNF and IL-1 $\beta$; Wang et al, 2012) that increase inflammation, and ultimately increase neuroexcitation (Stellwagen et al, 2005). There has been no attempt to isolate the signal downstream of TLR4 that is responsible for the adverse effects of opioids.

\section{solTNF is a Key Proinflammatory Product of TLR4 Signaling}

The present set of experiments tested the hypothesis that TLR4 contributes to morphine tolerance via modulation of inflammatory signaling and glutamate homeostasis in a solTNF-dependent manner. TNF is a type 1 transmembrane protein that naturally exists in two forms: the more common form, tmTNF, and the less abundant form, solTNF, which is formed after cleavage of tmTNF by ADAM17/TNF- $\alpha$ Converting Enzyme (TACE) (Kriegler et al, 1988). solTNF signals primarily through TNFRI, whereas tmTNF signals through both TNFRI and TNFRII. As TNFRII signaling is protective against glutamate excitotoxicity (Marchetti et al, 2004), and enhanced glutamatergic signaling contributes to opioid tolerance, we predicted that decreasing TNFRI signaling by selectively sequestering solTNF (while preserving TNFRII signaling) would prevent tolerance to morphine. Here, we manipulated PAG solTNF sequestration using a lentivirus and brain-permeant DN-TNF, and examined the impact on tolerance, cytokine release, and key elements of glutamate homeostasis.

\section{PAG Cytokines and TLR4 mRNA are Increased by Chronic Morphine}

In agreement with our previous findings (Eidson and Murphy, 2013), we show that tolerance to morphine developed rapidly. Indeed, systemic administration of one $\mathrm{ED}_{50}$ dose of morphine for 3 days is sufficient to induce tolerance as indicated by a threefold rightward shift in the morphine dose-response curve. Development of morphine tolerance is paralleled by increased gene expression of three major proinflammatory factors in the PAG: TLR4, TNF, and IL-1 $\beta$, indicating that chronic morphine induces inflammation within the PAG and primes PAG glia to overrespond to subsequent morphine challenges by increasing gene expression of the receptor substrate for opioid-mediated inflammation (TLR4; Wang et al, 2012). Although the increase in vlPAG TNF was not significant in the current study, and is in contrast to what has been noted in the spinal cord (Shen et al, 2011a), TNF is found in very low concentration in the CNS (femtomolar to picomolar range), and binds with high affinity to a relatively small number of receptors (Peterson et al, 1998). In vitro, TNF protein is significantly increased by the TLR4 agonist LPS in a TACE-dependent manner at 30 min following LPS application (von Maltzan et al, 2012). In vivo, acute intrathecal morphine analgesia is reduced by TNF within 5 min of morphine administration (Hutchinson et al, 2008), suggesting that morphine leads to the quick release of solTNF protein via TACE-mediated cleavage of tmTNF. As TNF is primarily responsible for initiating the 

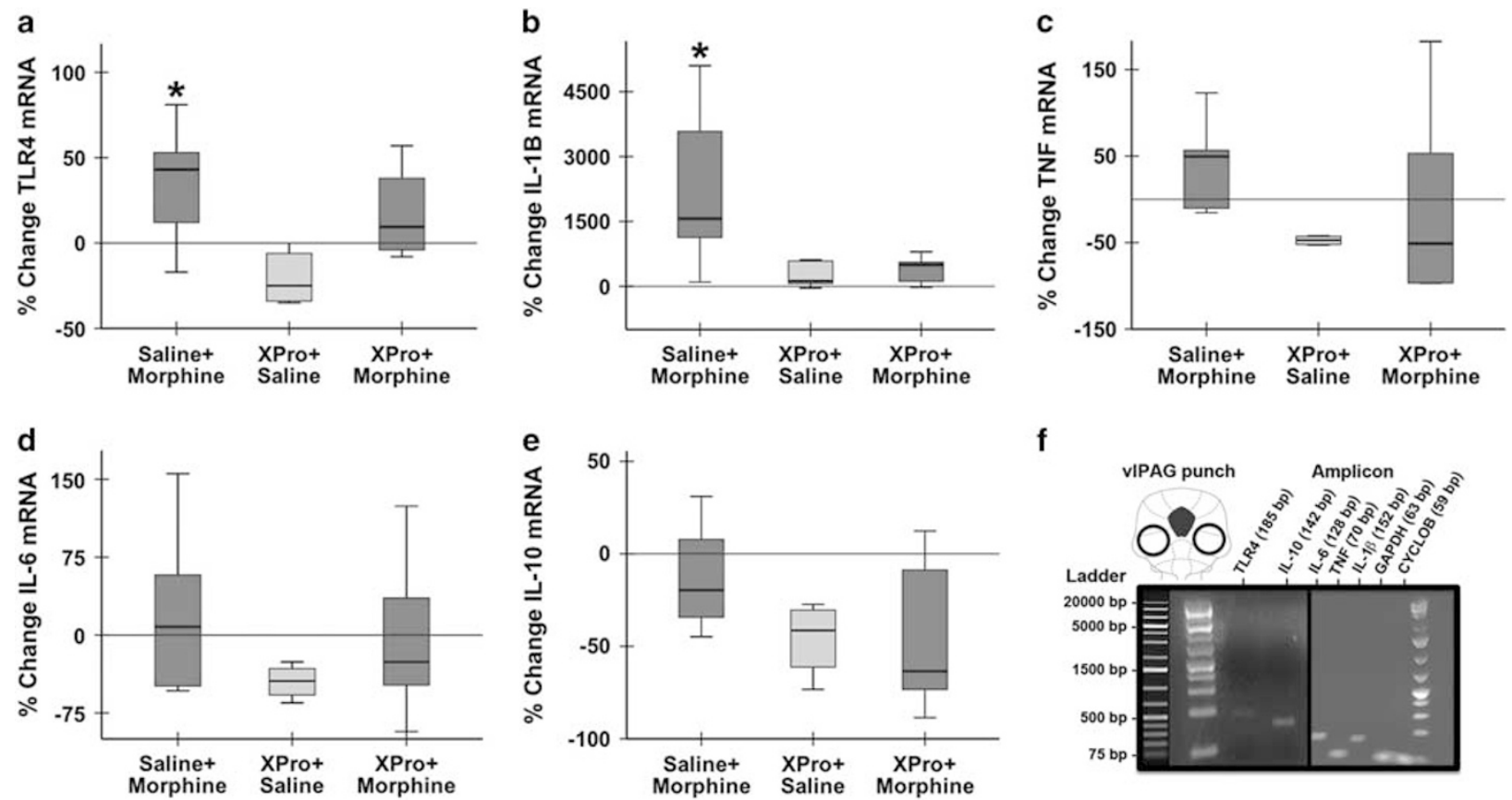

Figure 4 Summary of cytokine and Toll-like receptor 4 (TLR4) mRNA in the ventrolateral periaqueductal gray (VIPAG) following chronic systemic XProl595 and morphine. Systemic administration of XProl 595 prevents morphine induced increases in vIPAG interleukin- I $\beta$ (IL- I $\beta$ ) and TLR4 mRNA. (a) TIr4, (b) II / $\beta$, (c) Tnf, (d) II6, and (e) III 0 mRNA levels relative to the housekeeping gene Gapdh in the vIPAG of rats treated with Saline+Morphine, XPro +Saline, and XPro+Morphine. Data are represented as median \% change from Vehicle+Saline controls; $n=5$ per group. (f) All primer pairs produced amplicons of appropriate size as compared with the control DNA ladder. Representative schematic of vIPAG tissue punch location (top left). PCR amplicons from each gene of interest were run on a $2 \%$ agarose gel (main photomicrograph) to demonstrate primer specificity. All genes localized to regions appropriate to their predicted size. $* p<0.05$.

production of other proinflammatory cytokines (DeLeo et al, 2004), morphine signaling through TLR4 may induce rapid cleavage of tmTNF to solTNF protein and stimulate the production of IL- $1 \beta$ and TLR4 mRNA to modulate glutamate homeostasis. Our findings suggest that TACE inhibition may preserve morphine analgesia and prevent tolerance development in the vlPAG by preventing TLR4-mediated solTNF signaling. The PAG, along with the RVM and spinal cord, form the descending pain modulatory circuit, and all three regions are critical neural substrates for the analgesic effects of morphine. In the present study, we focused on TLR4mediated neuroinflammation within the PAG; however, similar neuroinflammatory spinal cord responses have been implicated in the development of morphine tolerance (Raghavendra et al, 2002).

\section{Inhibition of solTNF Prevents Tolerance to Morphine}

Systemically administered XPro1595 was efficiently transported to midbrain tissue, and normalized vlPAG TNF, IL-1 $\beta$, and TLR4 mRNA levels, effectively preserving morphine efficacy following chronic exposure. These data are the first to establish a role for TNF in opiate tolerance, and are the first to identify solTNF as a critical proinflammatory factor mediating opioid tolerance development. Currently, there are FDA-approved non-selective biological inhibitors that sequester both forms of TNF (solTNF and tmTNF), but these drugs have been associated with neurological deficits and demyelinating disease in patients who were previously neurologically normal (Seror et al,
2013), indicating the critical need to differentiate the actions of the two TNF isoforms.

\section{PAG solTNF Signaling is Necessary for Changes in mRNA Induced by Chronic Systemic Morphine or PAG LPS}

Morphine tolerance, induced by systemic morphine administration, decreased astrocytic (GLT-1 and GLAST), but not neuronal (EAACI), glutamate transporter mRNA in the vlPAG, complementing our previous findings (Eidson and Murphy, 2013), and a vast literature demonstrating a role for glia (Watkins et al, 2005) and excitatory neurotransmission (McLemore et al, 1997) in morphine tolerance. Astrocytes are responsible for the majority of glutamate uptake in the CNS via GLT-1 and GLAST (Rothstein et al, 1996), thereby terminating glutamatergic signaling (Kanai et al, 1993). A significant increase in CSF glutamate and aspartate has been reported in morphine-tolerant humans (Wong et al, 2002), and morphine challenge increases glutamate in the CSF of morphine-tolerant rats (Tai et al, 2006). Increased glutamate uptake by GLT-1 attenuates morphine tolerance in mice (Nakagawa et al, 2001). Taken together with our results, these data suggest that a breakdown in astrocyte-mediated glutamate homeostasis significantly contributes to opioid tolerance. Chronic vlPAG LPS resulted in behavioral and molecular phenotypes that paralleled morphine-treated rats, suggesting that TLR4 signaling in the PAG opposes morphine analgesia by promoting neuroinflammation and disrupting the main source of glutamate reuptake in the 


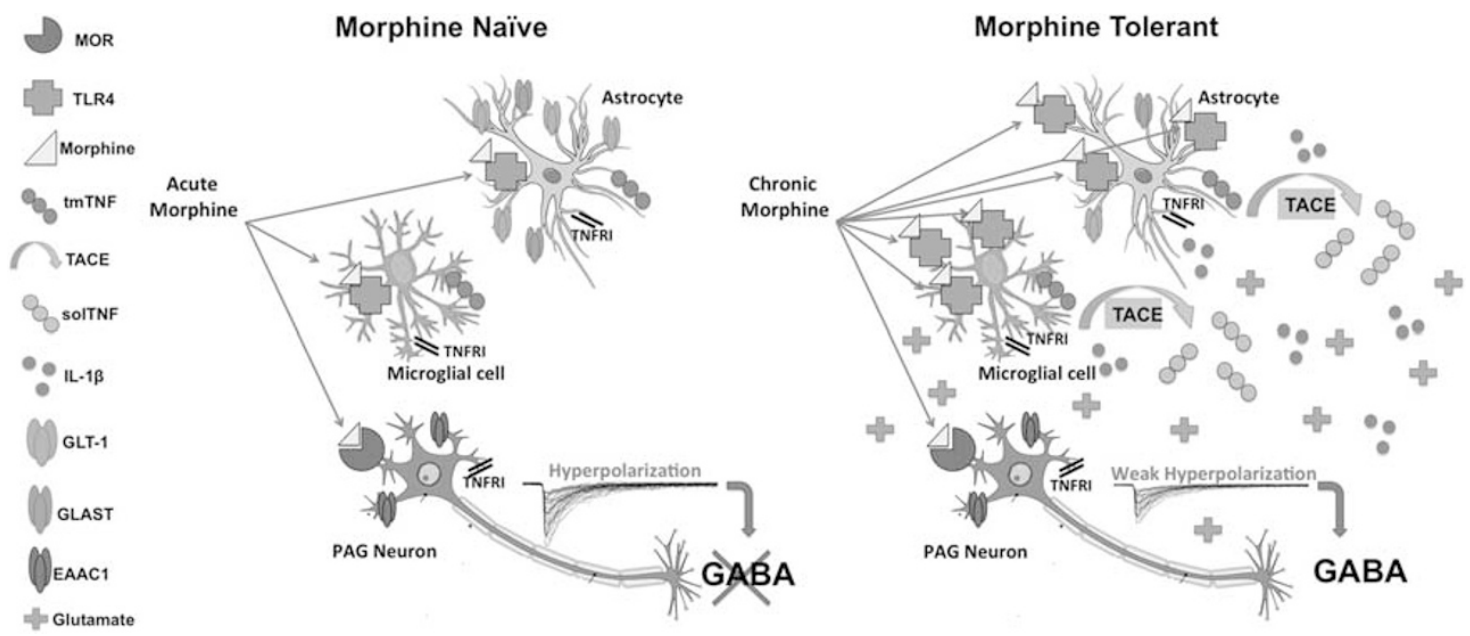

Figure 5 A schematic diagram illustrating our hypothesized model of soluble tumor necrosis factor (solTNF)-induced morphine tolerance development in the periaqueductal gray (PAG). Based on the major conclusions of our own data and the data of others, we hypothesize that chronic morphine binds to ventrolateral (VIPAG) Toll-like receptor 4 (TLR4) and leads to cleavage of transmembrane TNF (tmTNF) to solTNF by TNF-converting enzyme (TACE) to increase proinflammatory gene expression (TLR4, interleukin- I $\beta(\mathrm{IL}-\mathrm{I} \beta)$ ) and decrease astrocytic glutamate transporter mRNA (glutamate type I transporter (GLT-I) and glutamate aspartate transporter (GLAST)) in the vIPAG. These changes effectively increase the availability of glutamate in the synapse, thereby decreasing the ability of morphine to hyperpolarize GABAergic neurons. These changes associated with morphine tolerance prevent morphine from initiating signaling through the descending analgesic circuit. A full color version of this figure is available at the Neuropsychopharmacology journal online.

CNS. Sequestration of vlPAG solTNF normalized the morphine- and endotoxin-induced changes in glutamate transporter mRNA and prevented morphine tolerance, suggesting that solTNF signaling mediates the effects of TLR4.

\section{A Proposed Inflammatory Model of Morphine Tolerance}

Morphine and other opioids bind to neuronal mu opioid receptors (MORs) (Pasternak and Pan, 2013) and elicit analgesia, in part, by hyperpolarization of GABAergic neurons (Basbaum and Fields, 1978; Lau and Vaughan, 2014). Indeed, opioids have a direct inhibitory effect on most MOR-expressing neurons (Vaughan et al, 1997), including those in the descending analgesic circuit (eg, PAG) (Vaughan et al, 1997). Based on our current findings, we propose that morphine binds to neuronal MOR as well as glial TLR4 in the PAG, and that concurrent activity at these receptors modulates the analgesic efficacy of morphine via two opposing mechanisms: (i) opiate binding at MOR results in hyperpolarization of GABAergic neurons and induction of opiate analgesia; and (ii) opiate binding at glial TLR4 leads to increased vlPAG solTNF signaling that simultaneously promotes inflammation and disrupts the ability of astrocytes to scavenge excess glutamate, counteracting MOR-mediated hyperpolarization of GABAergic neurons to promote morphine tolerance. Key tenants of our proposed model are illustrated in Figure 5.

\section{CONCLUSIONS}

Our results support the use of solTNF sequestration peptides such as XPro1595 as a potential adjunct to opioid therapy. As tmTNF is the TNF ligand that signals through TNFRII in physiological conditions, and TNFRII is protective against glutamate excitotoxicity (Marchetti et al, 2004), anti-solTNF treatment is likely to be a dually beneficial countermeasure to
Table I Effective Doses for Morphine by Group

\begin{tabular}{|c|c|c|c|}
\hline Condition & $n$ & $E D_{50}(\mathrm{mg} / \mathrm{kg})$ & $\begin{array}{l}95 \% \text { Confidence } \\
\text { interval }\end{array}$ \\
\hline Lenti GFP+Saline & 4 & 2.50 & $1.86-3.06$ \\
\hline Lenti GFP+Morphine & 10 & 7.47 & $6.90-8.03$ \\
\hline Lenti DN-TNF+Saline & 5 & 3.93 & $2.97-4.88$ \\
\hline Lenti DN-TNF+Morphine & 10 & 2.66 & $2.14-3.18$ \\
\hline \multicolumn{4}{|l|}{$F_{(3,195)}=49.64 ; p<0.0001$} \\
\hline Lenti GFP+PAG Saline & 6 & 2.37 & $2.05-2.70$ \\
\hline Lenti GFP+PAG LPS & 4 & 6.00 & $5.42-6.58$ \\
\hline Lenti DN-TNF+PAG Saline & 6 & 3.00 & $2.61-3.40$ \\
\hline Lenti DN-TNF+PAG LPS & 9 & 3.26 & $2.90-3.62$ \\
\hline \multicolumn{4}{|l|}{$F_{(3,167)}=37.31 ; p<0.0001$} \\
\hline Vehicle+Saline & 8 & 2.30 & $1.99-2.60$ \\
\hline Vehicle+Morphine & 11 & 7.35 & $6.82-7.88$ \\
\hline XPro+Saline & 8 & 3.18 & $2.69-3.67$ \\
\hline XPro+Morphine & 11 & 2.25 & $1.86-2.65$ \\
\hline$F_{(3,258)}=87.31 ; p<0.0001$ & & & \\
\hline
\end{tabular}

Abbreviations: DN-TNF, dominant-negative TNF; ED 50 , effective dose 50\%; GFP, green fluorescent protein; LPS, lipopolysaccharide; PAG, periaqueductal gray; TNF, tumor necrosis factor.

the opioid-induced neuroexcitability known to contribute to tolerance (Trujillo and Akil, 1991; McLemore et al, 1997). As the mechanisms underlying hyperalgesia (DeLeo et al, 2004) are strikingly similar to the mechanisms underlying opioid tolerance development, these data suggest that selective antisolTNF biologics could complement opioid therapy in the clinic by suppressing nociceptive signals at the site of injury and in the spinal cord, as well as preserving morphine analgesic efficacy by preventing vlPAG glia activation. 


\section{FUNDING AND DISCLOSURE}

The authors declare no conflict of interest.

\section{ACKNOWLEDGMENTS}

David Sinkiewicz provided essential qPCR assistance. CJ Barnum provided XPro1595 consultation. Morphine sulfate, $(+)$-morphine, and (+)-naloxone were kindly provided by the National Institute on Drug Abuse (NIDA) drug supply program. XPro1595 was generously provided by David Szymkowski (Xencor) and RJ Tesi (FPRT Bio). National Institutes of Health grant DA16272 awarded to AZM supported this work. Sigma Xi Scientific Research Society Grant-In-Aid of Research, Georgia State University College of Arts and Sciences Dissertation Grant Award, and Georgia State University Center for Neuromics award to LNE supported this work. Contributions by LJY and KI were supported by funding provided by Office of Research Infrastructure Programs/OD P51OD11132 (to Yerkes National Primate Research Center). Viral vector production was supported, in part, by the Viral Vector Core of the Emory Neuroscience NINDS Core Facilities grant, P30NS055077. MGT is an ex-employee of Xencor, but does not hold significant financial stake in Xencor or FPRT Bio and is not a consultant.

\section{REFERENCES}

Bachtell R, Hutchinson MR, Wang X, Rice KC, Maier SF, Watkins LR (2015). Targeting the Toll of drug abuse: the translational potential of toll-like receptor 4. CNS Neurol Disord Drug Targets 14: 692-699.

Basbaum AI, Fields HL (1978). Endogenous pain control mechanisms: review and hypothesis. Ann Neurol 4: 451-462.

DeLeo JA, Tanga FY, Tawfik VL (2004). Neuroimmune activation and neuroinflammation in chronic pain and opioid tolerance/ hyperalgesia. Neuroscientist 10: 40-52.

Dowell D, Haegerich TM, Chou R (2016). CDC Guideline for Prescribing Opioids for Chronic Pain-United States, 2016. MMWR Recomm Rep 65: 1-49.

Eidson LN, Murphy AZ (2013). Blockade of Toll-like receptor 4 attenuates morphine tolerance and facilitates the pain relieving properties of morphine. J Neurosci 33: 15952-15963.

Fine SM, Angel RA, Perry SW, Epstein LG, Rothstein JD, Dewhurst $S$ et al (1996). Tumor necrosis factor alpha inhibits glutamate uptake by primary human astrocytes. Implications for pathogenesis of HIV-1 dementia. J Biol Chem 271: 15303-15306.

Gulur P, Williams L, Chaudhary S, Koury K, Jaff M (2014). Opioid tolerance-a predictor of increased length of stay and higher readmission rates. Pain Physician 17: E503-E507.

Hargreaves K, Dubner R, Brown F, Flores C, Joris J (1988). A new and sensitive method for measuring thermal nociception in cutaneous hyperalgesia. Pain 32: 77-88.

Heo YJ, Oh HJ, Jung YO, Cho ML, Lee SY, Yu JG et al (2011). The expression of the receptor for advanced glycation end-products (RAGE) in RA-FLS is induced by IL-17 via Act-1. Arthritis Res Ther 13: R113.

Hutchinson MR, Bland ST, Johnson KW, Rice KC, Maier SF, Watkins LR (2007). Opioid-induced glial activation: mechanisms of activation and implications for opioid analgesia, dependence, and reward. ScientificWorld J 7: 98-111.

Hutchinson MR, Coats BD, Lewis SS, Zhang Y, Sprunger DB, Rezvani N et al (2008). Proinflammatory cytokines oppose opioid-induced acute and chronic analgesia. Brain Behav Immun 22: $1178-1189$.
Inoue $\mathrm{K}$, Terashima $\mathrm{T}$, Nishikawa $\mathrm{T}$, Takumi $\mathrm{T}$ (2004). Fezl is layer-specifically expressed in the adult mouse neocortex. Eur $J$ Neurosci 20: 2909-2916.

Kanai Y, Smith CP, Hediger MA (1993). The elusive transporters with a high affinity for glutamate. Trends Neurosci 16: $365-370$

Kosek E, Altawil R, Kadetoff D, Finn A, Westman M, Le Maitre E et al (2015). Evidence of different mediators of central inflammation in dysfunctional and inflammatory pain-interleukin-8 in fibromyalgia and interleukin-1 beta in rheumatoid arthritis. J Neuroimmunol 280: 49-55.

Kriegler M, Perez C, DeFay K, Albert I, Lu SD (1988). A novel form of $\mathrm{TNF} /$ cachectin is a cell surface cytotoxic transmembrane protein: ramifications for the complex physiology of TNF. Cell 53: $45-53$.

Lau BK, Vaughan CW (2014). Descending modulation of pain: the GABA disinhibition hypothesis of analgesia. Curr Opin Neurobiol 29C: $159-164$

Loyd DR, Morgan MM, Murphy AZ (2008). Sexually dimorphic activation of the periaqueductal gray-rostral ventromedial medullary circuit during the development of tolerance to morphine in the rat. Eur J Neurosci 27: 1517-1524.

Marchetti L, Klein M, Schlett K, Pfizenmaier K, Eisel UL (2004). Tumor necrosis factor (TNF)-mediated neuroprotection against glutamate-induced excitotoxicity is enhanced by N-methyl-Daspartate receptor activation. Essential role of a TNF receptor 2mediated phosphatidylinositol 3-kinase-dependent NF-kappa B pathway. J Biol Chem 279: 32869-32881.

McCoy MK, Ruhn KA, Martinez TN, McAlpine FE, Blesch A, Tansey MG (2008). Intranigral lentiviral delivery of dominantnegative TNF attenuates neurodegeneration and behavioral deficits in hemiparkinsonian rats. Mol Ther 16: 1572-1579.

McLemore GL, Kest B, Inturrisi CE (1997). The effects of LY293558, an AMPA receptor antagonist, on acute and chronic morphine dependence. Brain Res 778: 120-126.

Nakagawa T, Ozawa T, Shige K, Yamamoto R, Minami M, Satoh M (2001). Inhibition of morphine tolerance and dependence by MS-153, a glutamate transporter activator. Eur J Pharmacol 419: $39-45$.

Ogoshi F, Yin HZ, Kuppumbatti Y, Song B, Amindari S, Weiss JH (2005). Tumor necrosis-factor-alpha (TNF-alpha) induces rapid insertion of $\mathrm{Ca}^{2+}$-permeable alpha-amino-3-hydroxyl-5-methyl4-isoxazole-propionate (AMPA)/kainate $(\mathrm{Ca}-\mathrm{A} / \mathrm{K})$ channels in a subset of hippocampal pyramidal neurons. Exp Neurol 193: 384-393.

Pasternak GW, Pan YX (2013). Mu opioids and their receptors: evolution of a concept. Pharmacol Rev 65: 1257-1317.

Peterson PK, Molitor TW, Chao CC (1998). The opioid-cytokine connection. J Neuroimmunol 83: 63-69.

Raghavendra V, Rutkowski MD, DeLeo JA (2002). The role of spinal neuroimmune activation in morphine tolerance/hyperalgesia in neuropathic and sham-operated rats. J Neurosci 22: 9980-9989.

Rothstein JD, Dykes-Hoberg M, Pardo CA, Bristol LA, Jin L, Kuncl RW et al (1996). Knockout of glutamate transporters reveals a major role for astroglial transport in excitotoxicity and clearance of glutamate. Neuron 16: 675-686.

Seror R, Richez C, Sordet C, Rist S, Gossec L, Direz G et al (2013). Pattern of demyelination occurring during anti-TNF-alpha therapy: a French national survey. Rheumatology (Oxford) 52: 868-874.

Shen $\mathrm{CH}$, Tsai RY, Shih MS, Lin SL, Tai YH, Chien CC et al (2011a). Etanercept restores the antinociceptive effect of morphine and suppresses spinal neuroinflammation in morphinetolerant rats. Anesth Analg 112: 454-459.

Shen CH, Tsai RY, Tai YH, Lin SL, Chien CC, Wong CS (2011b). Intrathecal etanercept partially restores morphine's antinociception in morphine-tolerant rats via attenuation of the glutamatergic transmission. Anesth Analg 113: 184-190. 
Steed PM, Tansey MG, Zalevsky J, Zhukovsky EA, Desjarlais JR, Szymkowski DE et al (2003). Inactivation of TNF signaling by rationally designed dominant-negative TNF variants. Science 301: $1895-1898$.

Stellwagen D, Beattie EC, Seo JY, Malenka RC (2005). Differential regulation of AMPA receptor and GABA receptor trafficking by tumor necrosis factor-alpha. J Neurosci 25: 3219-3228.

Sun J, Liu S, Mata M, Fink DJ, Hao S (2012). Transgene-mediated expression of tumor necrosis factor soluble receptor attenuates morphine tolerance in rats. Gene Therapy 19: 101-108.

Tai YH, Wang YH, Wang JJ, Tao PL, Tung CS, Wong CS (2006). Amitriptyline suppresses neuroinflammation and up-regulates glutamate transporters in morphine-tolerant rats. Pain 124: 77-86.

Trujillo KA, Akil H (1991). Inhibition of morphine tolerance and dependence by the NMDA receptor antagonist MK-801. Science 251: 85-87.

Vaughan CW, Ingram SL, Connor MA, Christie MJ (1997). How opioids inhibit GABA-mediated neurotransmission. Nature 390: 611-614. von Maltzan K, Tan W, Pruett SB (2012). Investigation of the role of TNF-alpha converting enzyme (TACE) in the inhibition of cell surface and soluble TNF-alpha production by acute ethanol exposure. PLoS One 7: e29890.

Wang X, Loram LC, Ramos K, de Jesus AJ, Thomas J, Cheng K et al (2012). Morphine activates neuroinflammation in a manner parallel to endotoxin. Proc Natl Acad Sci U S A 109: 6325-6330.

Watkins LR, Hutchinson MR, Johnston IN, Maier SF (2005). Glia: novel counter-regulators of opioid analgesia. Trends Neurosci 28: 661-669.

Wong CS, Chang YC, Yeh CC, Huang GS, Cherng CH (2002). Loss of intrathecal morphine analgesia in terminal cancer patients is associated with high levels of excitatory amino acids in the CSF. Can J Anaesth 49: 561-565.

Zalevsky J, Secher T, Ezhevsky SA, Janot L, Steed PM, O'Brien C et al (2007). Dominant-negative inhibitors of soluble TNF attenuate experimental arthritis without suppressing innate immunity to infection. J Immunol 179: 1872-1883.

Supplementary Information accompanies the paper on the Neuropsychopharmacology website (http://www.nature.com/npp) 